\title{
The Reliability and Validity of Subjective Notational Analysis in Comparison to Global Positioning System Tracking to Assess Athlete Movement Patterns
}

\author{
Sera N. Doğramaci, Mark L. Watsford, and Aron J. Murphy \\ Human Performance Laboratory, School of Leisure, Sport and Tourism, University of Technology Sydney, Kuring-Gai Campus, \\ Lindfield, New South Wales, Australia
}

\begin{abstract}
Doğramacı, SN, Watsford, ML, and Murphy, AJ. The reliability and validity of subjective notational analysis in comparison to GPS tracking to assess athlete movement patterns. $J$ Strength Cond Res 25(3): 852-859, 2011-Subjective notational analysis can be used to track players and analyse movement patterns during match-play of team sports such as futsal. The purpose of this study was to establish the validity and reliability of the Event Recorder for subjective notational analysis. A course was designed, replicating ten minutes of futsal match-play movement patterns, where ten participants undertook the course. The course allowed a comparison of data derived from subjective notational analysis, to the known distances of the course, and to GPS data. The study analysed six locomotor activity categories, focusing on total distance covered, total duration of activities and total frequency of activities. The values between the known measurements and the Event Recorder were similar, whereas the majority of significant differences were found between the Event Recorder and GPS values. The reliability of subjective notational analysis was established with all ten participants being analysed on two occasions, as well as analysing five random futsal players twice during match-play. Subjective notational analysis is a valid and reliable method of tracking player movements, and may be a preferred and more effective method than GPS, particularly for indoor sports such as futsal, and field sports where short distances and changes in direction are observed.
\end{abstract}

KEY WorDS GPS, player tracking, video analysis, futsal

Address correspondence to Sera N. Doğramac1, sera.dogramaci@uts.edu.au. 25(3)/852-859

Joumal of Strength and Conditioning Research

(C) 2011 National Strength and Conditioning Association

\section{INTRODUCTION}

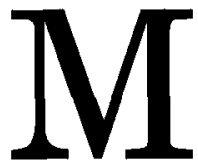

ovement analysis is important for understanding the physiological and technical demands associated with field and court team sports, and assisting coaches with the designing of training programs that may improve match performance $(4,7,9,18,25)$. Previous time-motion analysis studies have been conducted in Australian Rules Football $(2,19,25)$, basketball (1), field hockey $(23,24)$, rugby union $(13,14,18)$, soccer $(8,9,20,22)$, and more recently in futsal $(3,4,5,10,15)$. Tracking athletes involved in these sports may be difficult where fast, agile movements are performed, with changes in direction and collision of players (6).

Time-motion analysis provides a platform to analyze the movement patterns of athletes participating in various sports. This information is particularly important to understand player loading and intensity requirements of athletic endeavors, and to detect the predominant energy system in use and the onset of fatigue (4). Furthermore, changes in performance can be detected between different periods or halves of the game, throughout the season, by playing position, level of competition, or between individuals and teams (10).

Various notational motion analysis methods have included video-based tracking, computer-based tracking, and the use of electronic transmitters $(9,19)$. Video-based tracking involves videotaping players and analyzing the movement patterns either manually or with the aid of a computer postmatch $(7,13)$. Computer-based tracking relies on ground markings and reference points that translate to markers on a miniaturized, calibrated version of the playing field (19). Although each method uses different techniques, they are fundamentally measuring the same purpose, namely, movement patterns.

Most motion analysis methods, including video and computer-based tracking, are fairly labor intensive, where the analysis is often performed postmatch with only 1 player being tracked at a time. In recent times, global positioning system units (GPS) have been used to objectively quantify the movement demands of outdoor sports. Global positioning system has made player tracking more convenient and efficient 
than other methods because data are recorded in real time and multiple players can be tracked at any time. However, this method relies on the units having a clear view of the sky to pick up satellite signals $(16,17,19)$. Although this has resulted in a major shift in tracking methodologies for outdoor sports, such technology is not practical for use in indoor sports such as basketball, handball, water polo, or futsal.

As futsal is a developing sport in certain countries, a detailed understanding of the physical requirements would certainly aid in the improvement of match performance. There are currently few studies reporting the match demands of futsal that rely on subjective notational analysis $(4,5,11,15)$; therefore, the purpose of this study was to establish the validity and reliability of a method for subjective notational analysis. It was hypothesized that there would be no differences between data recorded via subjective notational analysis and criterion values. This hypothesis was tested using a range of locomotor activities typically encountered during court sports. Further, given the reported discrepancies in GPS data $(12,19)$, it was hypothesized that there may be significant differences between data collected using GPS and the criterion values for high-intensity variables, but no differences for total or low-intensity variables.

\section{MeTHODS}

\section{Experimental Approach to the Problem}

To assess the validity of the subjective notational analysis system, subjects completed a course consisting of activities typically seen in court sports. The distance and frequency results obtained from subjective notational analysis were compared with criterion values and GPS values. Further, by analyzing subjects twice on the same course and during a match, the reliability of the subjective notational analysis system used was assessed.

\section{Subjects}

Ten subjects were recruited for this study. They were all healthy, active, male university students (26.6 \pm 6.93 years, $184.9 \pm 6.95 \mathrm{~cm}$, and $85.5 \pm$ $12.7 \mathrm{~kg}$ ) who participated in a variety of sports including football, basketball, rugby union, rugby league, and tennis. Written informed consent was obtained from all subjects before conducting the study. The University of Technology, Sydney Human Research and Ethics Committee approved the methodology and procedures used in this study.

\section{Procedures}

Validity Analysis. Based on unpublished match-analysis data collected by the present research team, 10 courses were designed to replicate 10 minutes of specific futsal movement patterns. The average effort duration, frequency of change in locomotor activity, and nature of directional change were all made as specific to the movement patterns encountered during this high-intensity court sport. Having 10 different courses meant that each of the subjects was required to follow a different course from each other. A $15-\mathrm{m} \times 20-\mathrm{m}$ grid was marked out on a flat outdoor surface with 6 colored flags labeled 'A' through ' $F$ ' set at various points. The distance between each flag was measured with a tape measure, resulting in a total of 15 varying distances as shown in Figure 1 and Table 1. A mini DV camera (Panasonic, Osaka, Japan) was set up on a tripod $15 \mathrm{~m}$ away from one side of the course, at a height of $5 \mathrm{~m}$, which allowed the entire grid to be captured.

Subjects were screened for past and current injury history and were requested to refrain from performing exhaustive exercise in the 24 hour preceding the testing session. Subjects attended their testing session one at a time. Each participant was equipped with an SPI Elite Global Positioning System Unit (GPSports Systems, Canberra, Australia), which allowed a comparison of GPS measurements to the criterion distances already measured when designing the course, along with a comparison to notational analysis measurements.

The researcher commenced the test by calling out the first action subjects were to undertake and to which flag they were to proceed to. Each action and direction thereafter was called out before the participants finished their current action. For example, the researcher may have instructed the subject to 


\begin{tabular}{|c|c|c|c|c|c|}
\hline Path & $\begin{array}{c}\text { Distance } \\
(\mathrm{m})\end{array}$ & Path & $\begin{array}{l}\text { Distance } \\
(\mathrm{m})\end{array}$ & Path & $\begin{array}{c}\text { Distance } \\
(\mathrm{m})\end{array}$ \\
\hline $\begin{array}{l}A-B \\
A-C \\
A-D \\
A-E \\
A-F\end{array}$ & $\begin{array}{r}2.50 \\
11.18 \\
10.63 \\
3.16 \\
10.05\end{array}$ & $\begin{array}{l}B-C \\
B-D \\
B-E \\
B-F \\
C-D\end{array}$ & $\begin{array}{r}10.31 \\
12.62 \\
5.59 \\
10.59 \\
13.34\end{array}$ & $\begin{array}{l}C-E \\
C-F \\
D-E \\
D-F \\
E-F\end{array}$ & $\begin{array}{r}13.60 \\
20.88 \\
9.43 \\
18.38 \\
9.22\end{array}$ \\
\hline
\end{tabular}

run to A, jog backward to C, walk to D, stand for 3 seconds at $\mathrm{B}$, and so on, until the course was completed. Subjects were required to travel as close as possible to the base of the flag, placing their foot next to the flagstick before continuing on to the proceeding flag. They were also instructed to make hand contact with the top of the flag, rather than moving around the flag itself, to complete the actual distance between markers. Each participant was tracked by the video camera from the commencement of their course, until completion, with the audio function muted so the analyzer would not be able to hear the actions on the video playback. The notational analyzer was not aware of the various course designs to increase the validity of the study, and thus a different researcher instructed the subjects through the course.

The digital video footage from the study was transferred onto videotape to enable analysis. The researcher tracked each participant, and movements were noted using a console and program called 'Event Recorder' (ER) designed by KB Technologies (Sydney, Australia). This console permitted the analysis of 6 movement velocities. The identification of the speed of each locomotor activity was based on the researcher's subjective judgment, with the velocities and descriptions of activities selected from the methodologies of several researchers in other team sports such as in $(4,14,18,20,23,24)$. These were as follows:

Standing: $0 \mathrm{~m} \cdot \mathrm{s}^{-1}$, no visible movement of the feet.

Walking: $1 \mathrm{~m} \cdot \mathrm{s}^{-1}$, forward and backward movement with at least 1 foot in contact with the ground.

Jogging: $3 \mathrm{~m} \cdot \mathrm{s}^{-1}$, nonpurposeful slow running with low knee lift, involving a flight phase and minimal arm swing.

Running: $5 \mathrm{~m} \cdot \mathrm{s}^{-1}$, purposeful running with a higher knee lift and more pronounced arm swing than jogging.

Sprinting: $7 \mathrm{~m} \cdot \mathrm{s}^{-1}$, purposeful running with maximal effort.

Sideways/backwards: $3 \mathrm{~m} \cdot \mathrm{s}^{-1}$, lateral movement or backward jogging requiring effort and shuffling of the feet.

Each locomotor activity was timed to 0.1 seconds, and the total duration for each activity was calculated. The distance covered in each locomotor activity was calculated using the total duration of each activity by the speed assigned to it. Total frequency of activities was also computed to be able to calculate effort distances and durations. The raw (absolute) values were also converted into relative (percentage) values to enable a greater standardized comparison. Because GPS measures instantaneous positional data and calculates velocity, it cannot determine the type of movement performed such as sideways and backward movement; these variables were grouped with the jogging variable to make comparisons.

Reliability Analysis. The reliability of the ER was analyzed with the trials performed by all 10 subjects being analyzed on the course on 2 occasions, with 1 week separating the first and second analyses. Additional reliability procedures were undertaken using match situations. Video footage obtained from a futsal match was used, where 5 random futsal players from the same team were analyzed for distance covered, effort duration, and frequency of locomotor activities over a 10-minute period. The same locomotor categories were analyzed as used for the validity analysis. One experienced researcher was used to analyze the video footage, thereby minimizing any interindividual variation in the measures.

\section{Statistical Analyses}

All results were collated and various statistical procedures were implemented using Statistical Package for Social Sciences $\left(\right.$ SPSS $^{\circledast}$, version 16.0). Descriptive statistics for all raw and relative variables were calculated and are reported as mean $\pm S D$. To determine any differences in measurements between the criterion, ER and GPS values calculated via notational analysis, one-way analyses of variance (ANOVA) was conducted on the distance and frequency variables. Post Hoc Bonferroni tests were conducted on significantly different data, to determine between which analysis methods the differences occurred. The data did not violate any assumptions in ANOVA. For the reliability analysis, paired-samples $t$-tests were undertaken on the primary dependent variables of distance and frequency for the course data, and the futsal match data. An alpha level of $p \leq 0.05$ was selected as the criterion for significance for all statistical procedures.

\section{Results}

\section{Validity Analysis}

Distance Variables. A significant difference was evident in total distance covered $(p<0.01)$ where the total distance derived from the GPS was lower than the criterion distance and the ER distance. Post hoc Bonferroni tests revealed that the differences occurred between the GPS and criterion $(p<0.01)$, and between GPS and the ER $(p<0.01$ ) (GPS distance $1,101.9 \pm 52.6 \mathrm{~m}$, criterion distance $1,260.5 \pm 61.6 \mathrm{~m}$, and $\mathrm{ER}$ distance $1,265.4 \pm 64.5 \mathrm{~m})$. When considering measures of accuracy, there was a typical error of measurement (TEM) of $25.2 \mathrm{~m}$ or $2.2 \%$ between the GPS and criterion, and $26.1 \mathrm{~m}$ or $2.1 \%$ between the ER and criterion. When divided into individual activities, several significant differences were observed in both raw and relative data. These can be observed in Table 2. 


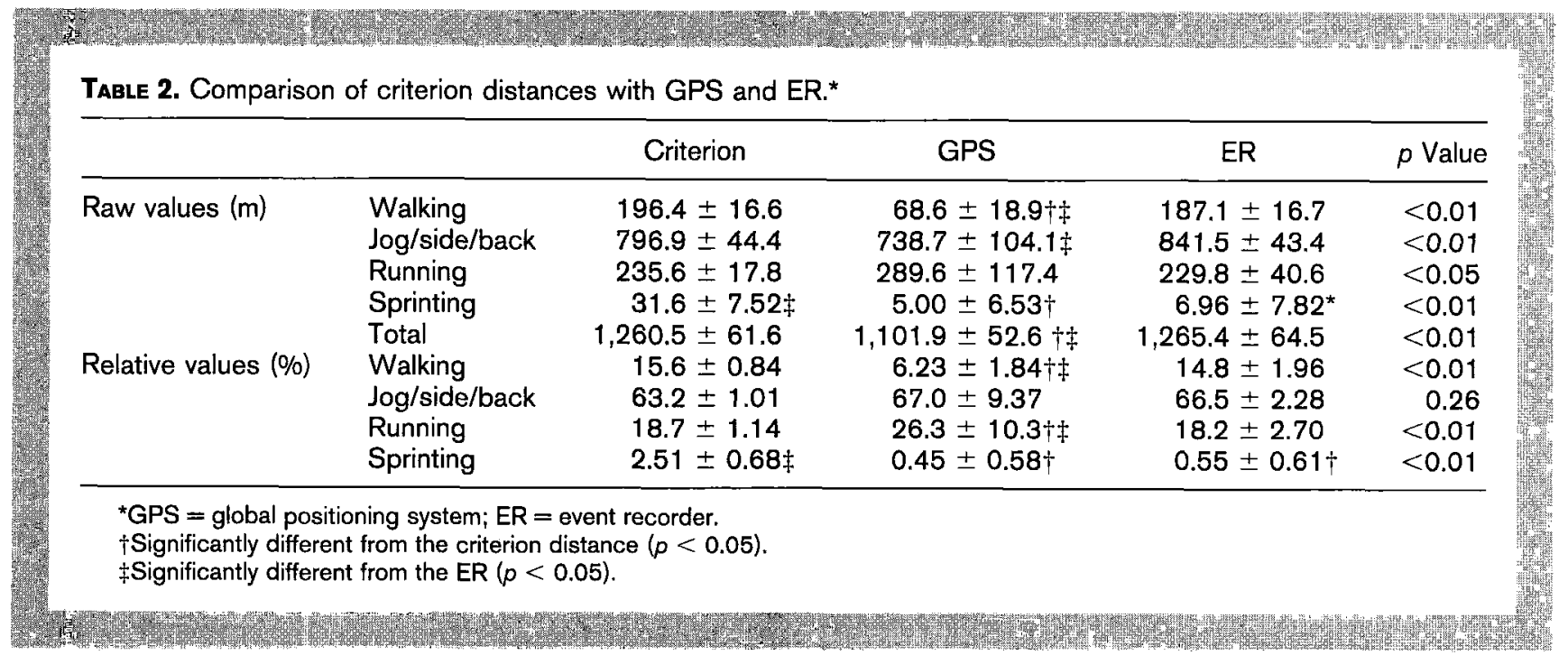

As evidenced in Table 2, the values between the criterion measurements and ER were similar, as confirmed with post hoc tests, with the exception of sprinting. The majority of significant differences occurred between the GPS and the criterion measurements, and between the GPS and ER. The GPS values generally underestimated the distance when compared with the criterion values and those calculated from the ER. When sprinting was observed, significant differences occurred between the criterion values and GPS (raw values $p<0.01$, relative values $p<0.01$ ), and also between the criterion values and ER (raw values $p<0.01$, relative values $p<0.01$ ).

Frequency Variables. Significant main effect differences were revealed between the groups when observing the total frequency of activities $(p<0.01)$, with the GPS calculating a greater number of activity occurrences than the criterion values and the ER (criterion value $128.0 \pm 6.32$; GPS value $189.0 \pm 19.2$; and ER value $133.9 \pm 8.97$ ). There was a difference of 61.0 actions or $32.3 \%$ between the GPS and the criterion values, with a TEM of 13.0 or $7.8 \%$. In contrast, the difference between the ER and criterion values was 5.9 actions or $4.4 \%$, with a TEM of 7.6 or $6.2 \%$, although this was not significantly different. Significant differences were observed between the individual variables that can be observed in Table 3 . The majority of GPS values were significantly higher than the criterion distance and $E R$ values; however, the raw and relative sprinting values from the GPS and the ER were significantly lower than the criterion value.

\section{Reliability Analysis}

Paired-samples $t$-tests were performed to compare the data obtained from trials 1 and 2 of the validity course. Further, paired-samples $t$-tests were used to compare the data from

TABLE 3. Comparison of criterion frequencies with GPS and ER.*

\begin{tabular}{llccrr}
\hline & & Criterion & GPS & ER & $p$ Value \\
\hline Raw values $(n)$ & Standing & $6.60 \pm 0.70$ & $9.00 \pm 4.92$ & $8.60 \pm 1.07$ & 0.17 \\
& Walking & $29.2 \pm 2.62$ & $55.9 \pm 12.4 \dagger$ & $35.8 \pm 8.04$ & $<0.01$ \\
& Jog/side/back & $71.8 \pm 3.71$ & $82.4 \pm 6.70 \dagger$ & $76.1 \pm 6.01$ & $<0.01$ \\
& Running & $17.9 \pm 1.66$ & $40.9 \pm 15.1 \dagger \ddagger$ & $12.1 \pm 5.32$ & $<0.05$ \\
& Sprinting & $2.50 \pm 0.53 \ddagger$ & $0.80 \pm 1.23 \dagger$ & $1.00 \pm 1.33 \dagger$ & 0.01 \\
& Total & $128.0 \pm 6.32$ & $189.0 \pm 19.2 \ddagger \dagger$ & $133.9 \pm 8.97$ & $<0.01$ \\
Relative values (\%) & Standing & $5.16 \pm 0.38$ & $4.76 \pm 2.23 \dagger$ & $6.42 \pm 0.75$ & 0.03 \\
& Walking & $22.8 \pm 2.01$ & $29.63 \pm 6.62 \dagger$ & $26.7 \pm 6.15$ & 0.04 \\
& Jog/side/back & $56.1 \pm 1.06$ & $43.6 \pm 1.92 \dagger \dagger$ & $56.8 \pm 4.05$ & $<0.01$ \\
& Running & $14.0 \pm 0.85$ & $21.6 \pm 7.62 \dagger \dagger$ & $9.04 \pm 3.58$ & $<0.01$ \\
& Sprinting & $1.95 \pm 0.39 \ddagger$ & $0.42 \pm 0.62 \dagger$ & $0.75 \pm 0.94 \dagger$ & $<0.01$ \\
\hline
\end{tabular}

*GPS $=$ global positioning system; $E R=$ event recorder.

$\dagger$ Significantly different from the criterion frequency $(p<0.05)$.

$\ddagger$ Significantly different from the ER $(p<0.05)$. 


\begin{tabular}{|c|c|c|c|c|}
\hline & & Trial 1 & Trial 2 & $p$ Value \\
\hline \multirow[t]{4}{*}{ Distance $(\mathrm{m})$} & Walking & $163.3 \pm 19.9$ & $181.1 \pm 19.8$ & 0.06 \\
\hline & Jog/side/back & $844.3 \pm 42.8$ & $841.5 \pm 43.4$ & 0.78 \\
\hline & Running & $297.3 \pm 34.0$ & $280.8 \pm 48.2$ & 0.70 \\
\hline & Sprinting & $7.24 \pm 8.60$ & $6.96 \pm 7.82$ & 0.65 \\
\hline \multirow[t]{5}{*}{ Duration (s) } & Standing & $39.1 \pm 3.03$ & $49.1 \pm 3.03$ & 0.06 \\
\hline & Walking & $163.3 \pm 19.9$ & $181.1 \pm 19.8$ & 0.78 \\
\hline & Jog/side/back & $281.4 \pm 14.3$ & $280.5 \pm 14.5$ & 0.07 \\
\hline & Running & $59.5 \pm 6.80$ & $56.2 \pm 9.63$ & 0.66 \\
\hline & Sprinting & $1.03 \pm 1.23$ & $0.99 \pm 1.12^{*}$ & 0.02 \\
\hline \multirow[t]{5}{*}{ Frequency $(n)$} & Standing & $7.30 \pm 1.49$ & $8.10 \pm 1.20$ & 0.14 \\
\hline & Walking & $34.5 \pm 7.26$ & $35.6 \pm 8.17$ & 0.12 \\
\hline & Jog/side/back & $74.1 \pm 5.24$ & $76.1 \pm 6.01$ & 0.23 \\
\hline & Running & $17.6 \pm 2.32$ & $15.3 \pm 4.52$ & 0.08 \\
\hline & Sprinting & $1.30 \pm 1.42$ & $1.00 \pm 1.33$ & 0.28 \\
\hline
\end{tabular}

*Significantly different from trial $1(p<0.05)$.

analysis 1 and analysis 2 of the futsal match. When considering the course using ER, the total distance covered between both trials was similar (trial $11,332.1 \pm 55.4 \mathrm{~m} \mathrm{v}$. trial $21,310.4 \pm 59.8, p=0.92$ ), with a TEM of $35.3 \mathrm{~m}$ (2.7\%). There was, however, a significant difference between the trials for total duration (trial $1544.3 \pm 15.1$ s v. trial $2567.9 \pm 16.3$ seconds, $p=0.02$ ), with a TEM of 18.1 seconds (3.3\%). There was no significant difference when observing the total frequency of activities between the trials (trial $1134.8 \pm 6.12 \mathrm{v}$. trial $2133.6 \pm 8.90, p=0.67$ ), with a TEM of $6.12(4.8 \%)$. Data displaying other relevant variables from the validity course variables are reported in Table 4.

Within the 10 minutes of futsal match analyzed, no significant differences were recorded for the total raw distance covered (trial 1 2,451.3 $\pm 480.2 \mathrm{~m}$ v. trial 2 2,474.2 $\pm 201.5 \mathrm{~m}$, TEM: $217.7 \mathrm{~m}$ [11.1\%]). There was no significant difference between the trials for the total duration of activities (trial 1

\begin{tabular}{|c|c|c|c|c|}
\hline & & Trial 1 & Trial 2 & $p$ Value \\
\hline \multirow[t]{5}{*}{ Distance $(\mathrm{m})$} & Walking & $611.1 \pm 208.0$ & $588.3 \pm 211.6$ & 0.11 \\
\hline & Jogging & $766.4 \pm 174.9$ & $766.4 \pm 172.2$ & 1.00 \\
\hline & Running & $716.5 \pm 165.1$ & $629.1 \pm 168.5$ & 0.54 \\
\hline & Sprinting & $69.3 \pm 35.1$ & $55.0 \pm 33.3$ & 0.26 \\
\hline & Side/back & $348.0 \pm 70.1$ & $435.3 \pm 82.9$ & 0.10 \\
\hline \multirow[t]{6}{*}{ Duration (s) } & Standing & $60.2 \pm 38.1$ & $69.3 \pm 42.4$ & 0.18 \\
\hline & Walking & $611.1 \pm 212.3$ & $588.3 \pm 211.6$ & 0.11 \\
\hline & Jogging & $255.5 \pm 58.3$ & $255.5 \pm 57.4$ & 1.00 \\
\hline & Running & $143.3 \pm 33.0$ & $125.8 \pm 33.7$ & 0.54 \\
\hline & Sprinting & $9.91 \pm 5.01$ & $7.86 \pm 4.75$ & 0.26 \\
\hline & Side/back & $111.6 \pm 13.1$ & $145.1 \pm 27.6$ & 0.08 \\
\hline \multirow[t]{6}{*}{ Frequency $(n)$} & Standing & $17.2 \pm 10.5$ & $18.2 \pm 10.3$ & 0.09 \\
\hline & Walking & $89.8 \pm 24.3$ & $101.2 \pm 29.7^{*}$ & 0.05 \\
\hline & Jogging & $103.2 \pm 23.7$ & $110.2 \pm 18.7$ & 0.10 \\
\hline & Running & $54.2 \pm 19.8$ & $66.8 \pm 15.3$ & 0.07 \\
\hline & Sprinting & $5.60 \pm 2.19$ & $5.60 \pm 2.70$ & 1.00 \\
\hline & Side/back & $72.4 \pm 14.9$ & $84.2 \pm 12.5$ & 0.16 \\
\hline
\end{tabular}

*Significantly different from trial $1(\rho<0.05)$. 
$1,191.5 \pm 242.7 \mathrm{~s}$ v. trial $21,191.9 \pm 233.1$ seconds, TEM: 19.5 seconds $[1.70 \%])$; however, there was a significant difference for the total frequency of activities $(p=0.02$, trial $1342.4 \pm$ 42.2 v. trial $2386.2 \pm 47.9$, TEM: 19.6, 5.00\%). Data displaying other significant differences within the futsal match can be found in Table 5 .

\section{Discussion}

The validity of notational analysis has been previously reported, with researchers examining predicted distances obtained from video footage against known distances, obtained from ground dimensions such as the goal line on a soccer field $(2,13,19)$. In the current study, this design was used for a course replicating 10 minutes of futsal match play, with known distances between each point providing a criterion measure. For both the validity and reliability components of this study, total distance and frequency were examined, along with individual locomotor activities within each variable. This is the first study to specifically compare a notational tracking method with a criterion measure and a GPS tracking device. The reliability of GPS has been previously reported $(12,19)$; however, because this technology continues to emerge as a favorable method of tracking movement patterns, there is still merit in notational tracking. Coutts and Duffield (12) reported that GPS devices were appropriate for the measurement of total distances and peak speeds; however, they lacked the ability to reliably report high-intensity activities. Edgecomb and Norton (19) similarly reported that relatively large errors are produced when small distances are observed; however, errors become relatively less as distances are increased. In the current study, when results obtained from notational tracking were compared with a criterion measure, similar outcomes were evident. The ER produced values comparable to a criterion measure, both of which were significantly different to GPS values. However, sprinting values were similar between the ER and GPS, and significantly different to the criterion. When considering these results, for all variables, with the exception of sprinting, subjective notational analysis appears to be methodologically sound for monitoring player movements. This is of particular importance because indoor sports rely on notational analysis in tracking the movement patterns of athletes. In terms of sprinting, it appears that ER and GPS methods of analysis possess the same amount of error.

In congruence with the hypothesis, the distance and frequency values between the criterion measurements and the ER were similar. Interestingly, there were several significant differences evident between the ER and GPS values, which were not expected. In each case, the ER produced higher levels of accuracy. Significant differences were also observed between the GPS values and the criterion values, where GPS tended to underestimate the values for distance including the total overall distance, and overestimate the actual values for frequency of activity.
It has been previously suggested that when small distances are observed (such as $200 \mathrm{~m}$ ), relatively large errors are produced, however, as distances are increased (such as $2 \mathrm{~km}$ ), the errors become relatively less (19). Therefore, it was anticipated that there would be some differences between individual locomotor activity values, with the largest errors occurring with the sprinting variables, because this only appeared 2 or 3 times in each course, with a maximum distance of $21 \mathrm{~m}$. Within the sprinting variable, the values obtained from the ER and GPS were similar, with both values being significantly lower than the criterion values. This included the raw and relative sprinting distances and frequencies. In the absence of being able to use GPS indoors, notational analysis provides a similar precision of measurement as GPS, as long as the identified limitations are considered and acknowledged.

Computer-based tracking and GPS have been known to overestimate the actual distance covered with TEMs of 9.3 and $5.5 \%$, respectively (19). In contrast, the GPS values obtained from this study produced a relative TEM of $2.2 \%$, suggesting that the values obtained in this study were similar to the known distances when considering the total values. Similarly, the distances derived from the ER in the validity component of this study were similar to the criterion distances, with a relative TEM of $2.1 \%$. The ER also produced a relative TEM of $3.1 \%$ for total duration, and $6.20 \%$ for total frequency of activities, whereas for GPS these values were 9.6 and $7.80 \%$, respectively. In congruence, various GPS devices have shown a good level of accuracy, producing a difference of $0.7-4.1 \%$ in total distance covered when compared with true values, and a coefficient of variation between 4.0 and 7.2 $\mathrm{m}$ (12). These results suggest that the ER is an excellent device to track movement patterns, and may in fact have a greater accuracy than GPS at certain speeds. Furthermore, the ER can be used to track distance, duration, and frequency of activities of athletes involved in outdoor sports and indoor sports, which GPS currently is unable to perform. This is particularly important for indoor sports such as futsal, basketball, handball, and water polo, where notational analysis is essential in developing a greater understanding of match demands, which can thereby assist in improving match performance.

The reliability of the ER was also tested where several significant differences were observed. These included the total duration of activities in the validity course where the value was higher in trial 2 than in trial 1, resulting in a TEM of $3.3 \%$ or 18.1 seconds. For the 40 -minute futsal match, the total frequency of activities was lower in trial 2 than in trial 1, with a TEM of $5.0 \%$ or 19.6 activities. A reliability study conducted by Duthie et al. (18) examining a notational analysis in a rugby union match demonstrated moderate to poor reliability for total duration of activities with a TEM of $5.8-11.1 \%$, and a TEM of $4.3-13.6 \%$ for frequency of individual activities, indicating good to poor reliability. Conceivably, the lower TEM's in this study may be because 
of the lower duration of the total time. Where Duthie et al. (18) conducted the reliability analysis over 80 minutes, the validity course in this study was approximately 10 minutes for all subjects, and the futsal match was 40 minutes; therefore, it should be expected that fewer errors would be produced in this study. In any case, the TEMs in this study indicate good to moderate reliability for notational analysis during the course duration and the futsal match, and an experienced analyzer would be able to carry out the analysis confidently, as hypothesized.

Although the GPS analysis tended to underestimate the actual values in the current results, previous studies have demonstrated that GPS and computer-based tracking generally overestimate actual values (19). Within GPS analysis, the error may be because of satellite tracking technology, whereas within computer-based tracking analysis, the error may arise from human error from slight movements of the mouse or mouse pen (19). The errors produced in the ER analysis may also be attributed to operator error, with discrepancies in the timing of the movement patterns along with the interpretation of the locomotor activities. In addition, while being given clear, audible instructions, subjects may have performed different movements to what they were instructed. For example, when told to sprint, an individual may not have actually been able to achieve a velocity associated with sprinting, and thus, the ER operator may have misinterpreted the movement pattern. This occurred as the analyzer was not aware of the instructions and interpreted the movements as viewed on the video.

Within the GPS analysis, a velocity is not measured until a player actually attains the predetermined criteria for that specific velocity. Although the course in the present study was designed to include sprinting, most subjects did not attain a sufficient velocity to be classed as sprinting for the GPS analysis. Such an example was subsequently categorized as running. With the subjective notational analysis, a movement is quantified over the initial parts of the movement before the actual velocity is attained, which may improve the accuracy of the movement categories by classing movements even if the speed is not actually attained. Therefore, if a participant appeared to have been performing maximal exertion, this would have been categorized as sprinting. This may explain the lack of difference between the ER and the GPS analysis for the sprinting variables, where the discrepancies between the criterion data and the data from the ER and GPS may have been related to the acceleration ability of the subjects rather than a methodological issue. Typically, the sprinting distances in many team sports are too short to allow for maximum velocity to be reached, with most sprints lasting less than 1 second $(12,26)$. This supports the notion that although athletes may be performing maximal exertion, their velocity may not be sufficient to be classed as 'sprinting' in various tracking devices, thereby omitting vital data (12). Clearly, alternate measures of high-intensity activity such as heart rate, blood lactate, or frequency of accelerations may be more appropriate when measuring high-intensity activity rather than relying solely on analyzing movement patterns.

Global positioning system has several advantages over notational analysis, in that data are collected and produced in real time (19), whereas the notational analysis is often performed postevent, is fairly labor intensive and can be monotonous, particularly when one researcher is conducting the analysis $(6,7,9)$. Furthermore, several GPS units are also able to collect heart rate data that can provide additional information on intensity (9). Other disadvantages of notational analysis include only being able to track 1 participant at a time, confusion for the analyzer caused by player congestion because of the unpredictability of movements, the entire playing area may not included in the camera's field of view and finally, using multiple cameras and computers next to the playing area may not be convenient (7).

However, the ER has several advantages over the use of GPS for player tracking. Firstly, the ER may be used indoors and outdoors, whereas GPS is currently limited to outdoor use. This enables notational analysis to be performed for indoor sports such as futsal, basketball, and handball. Secondly, GPS and computer-based tracking do not consider the type of movement being performed, neglecting movements such as sideways or backward movement. This could provide crucial information that may often be a key factor in performance, particularly when short distances are considered. However, in this study, sideways and backward movements were combined with jogging, in order to permit a comparison of notational analysis values and GPS values. Furthermore, the ER and computer-based tracking do not require player instrumentation; therefore, players can participate in matches without any interference from equipment, or potential injury in sports where contact may be involved (19) and opposing players and teams can be tracked as well (7). Further, there is no limit to the number of players analyzed per match. Some codes currently restrict the number of players permitted to wear the devices during matches, such as Australian Rules Football, whereas others prevent their use in matches entirely. Additionally, GPS and other equipment may often be expensive $(9,21)$, and some teams wanting to track their players may not be able to afford such equipment, in this case, notational analysis may be a more convenient and cost effective option (7). Finally, GPS devices take a few minutes to initialize, during which period erroneous data may be recorded (16). Although these data can be easily identified if players are asked to stand for a few minutes, this may not be practical during match play where researchers have limited control of the players (16).

Video analysis methods have tended to be considered the most valid system for tracking movement patterns in athletes $(18,19)$. The overall error between notational analysis, GPS and computer-based tracking when used by experienced analyzers is within acceptable limits $(12,19)$. With the increasing digital technology in performance analysis, tracking movement patterns in sports has undoubtedly been 
enhanced (7); however, researchers must consider the reliability of such measures. The reliability is dependent on the particular method used, the data entry procedure, the number of analyzers, and their experience (7). Despite these issues, notational analysis has been proven reliable in previous studies $(7,18,21)$, and the results of the current study suggest that the ER is a valid and reliable method of notational analysis in tracking player movements. In fact, notational analysis may be a preferred and more effective method for tracking than GPS, particularly for indoor sports such as futsal, and field and court sports where short distances and changes in direction are observed.

\section{Practical Applications}

This study proved that subjective notational analysis is a valid and reliable method for tracking movement patterns in athletes, even with the advanced and often expensive technology available today. The ER was able to measure accurately distance and frequency variables over a course with known distances, when compared with criterion measures and GPS values. Subjective notational analysis, as shown in this study, was in fact superior in accuracy in several variables when compared with GPS, and has the advantage of being able to be used indoors, where GPS is currently limited to outdoor use.

\section{REFERENCES}

1. Abdelkrim, NB, El Fazaa, S, and El Ati, J. Time-motion analysis and physiological data of elite under-19-year-old basketball players during competition. Br J Sports Med 41: 69-75, 2007.

2. Appleby, B and Dawson, B. Video analysis of selected game activities in Australian rules football. J Sci Med Sport 5: 129-142, 2002.

3. Barbero Álvarez, JC and Castagna, C. Activity patterns in professional futsal players using global position tracking system. $J$ Sports Sci Med 6: 208-209, 2007

4. Barbero-Álvarez, JC, Soto, V, and Granda, J. Effort profiling during indoor soccer competition. J Sports Sci 22: 500-501, 2004.

5. Barbero-Álvarez, JC, Soto, VM, Barbero-Álvarez, V, and Granda-Vera, J. Match analysis and heart rate of futsal players during competition. J Sports Sci 26: 63-73, 2008.

6. Barris, S. Automatic tracking and the analysis of human movement. Int J Perf Anal Sport 8: 102-113, 2008.

7. Barris, $S$ and Button, C. A review of vision-based motion analysis in sport. Sparts Med 38: 1025-1043, 2008.

8. Barros, RML, Misuta, MS, Menezes, RP, Figueroa, PJ, Moura, FA, Cunha, SA, Anido, R, and Leite, NJ. Analysis of the distances covered by first division Brazilian soccer players obtained with an automatic tracking method. I Sports Sci Med 6: 233-242, 2007.

9. Carling, C, Bloomfield, J, Nelsen, L, and Reilly, T. The role of motion analysis in elite soccer: Contemporary performance measurement techniques and work rate data. Sports Med 38: 839-862, 2008.

10. Castagna, C, D'Ottavio, S, and Abt, G. Activity profile of young soccer players during actual match play. $J$ Strength Cond Res 17 : 775-780, 2003.

11. Castagna, C, D'Ottavio, S, Vera, JG, and Alvarez, JC. Match demands of professional futsal: A case study. J Sci Med Sport 12: 490-494, 2009.

12. Coutts, AJ and Duffield, R. Validity and reliability of GPS devices for measuring movement demands of team sport. J Sci Med Spont 13: $133-135,2010$.

13. Deutsch, MU, Kearney, GA, and Rehrer, NJ. Time-motion analysis of professional rugby union players during match-play. J Sports $S C i$ 25: 461-472, 2007.

14. Docherty, D, Wenger, HA, and Neary, P. Time-motion analysis related to the physiological demands of rugby. J Hum Mov Stud 14: 269-277, 1988.

15. Doğramacı, SN and Watsford, ML. A comparison of two different methods for time-motion analysis in team sports. Int J Perf Anal Sport 6: 73-83, 2006.

16. Duncan, MJ, Badland, HM, and Mummery, WK Applying GPS to enhance understanding of transport-related physical activity. $J S C i$ Med Sport 12: 583-585, 2009.

17. Duncan, MJ, Mummery, WK, and Dascombe, BJ. Utility of global positioning system to measure active transport in urban areas. Med Sci Sports Exerc 39: 1851-1857, 2007.

18. Duthie, $G$, Pyne, $D$, and Hooper, $S$. The reliability of video based time motion analysis. J Hum Mov Stud 44: 259-272, 2003.

19. Edgecomb, SJ and Norton, KI. Comparison of global positioning and computer-based tracking systems for measuring player movement distance during Australian football. J Sci Med Sport 9: 25-32, 2006.

20. Mohr, M, Krustrup, $P$, and Bangsbo, J. Match performance of highstandard soccer players with special reference to development of fatigue. J Sports Sci 21: 519-528, 2003.

21. O'Donoghue, $P$. Sources of variability in time-motion data; Measurement error and within player variability in work-rate. Int J Perf Anal Sport 4: 42-49, 2004.

22. Rampinini, E, Impellizzeri, FM, Castagna, C, Coutts, AJ, and Wisloff, U. Technical performance during soccer matches of the Italian Serie A league: Effect of fatigue and competitive level. J Si Med Sport 12: 227-233, 2009.

23. Spencer, M, Lawrence, S, Rechichi, C, Bishop, D, Dawson, B, and Goodman, C. Time-motion analysis of elite field hockey, with special reference to repeated-sprint ability. J Sport Sci 22: 843-850, 2004.

24. Spencer, M, Rechichi, C, Lawrence, S, Dawson, B, Bishop, D, and Goodman, C. Time-motion analysis of elite field hockey during several games in succession: A tournament scenario. $J$ Sci Med Sport 8: 382-391, 2005.

25. Veale, JP, Pearce, AJ, and Carlson, JS. Player movement patterns in an elite junior Australian rules football team: An exploratory study. J Sci Med Sport 6: 254-260, 2007.

26. Young, W, McLean, B, and Ardagna, J. Relationship between strength qualities and sprinting performance. $J$ Sports Med Phys Fitness 35: 13-19, 1995. 


\section{Journal of Strength and Conditioning Research}

Title Details

Related Titles

= Alternative Media Edition (1)

\section{Lists}

Marked Titles (0)

\section{Search History}

Journal of Strength and Conditioning Research

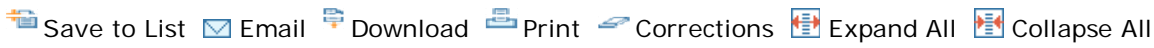

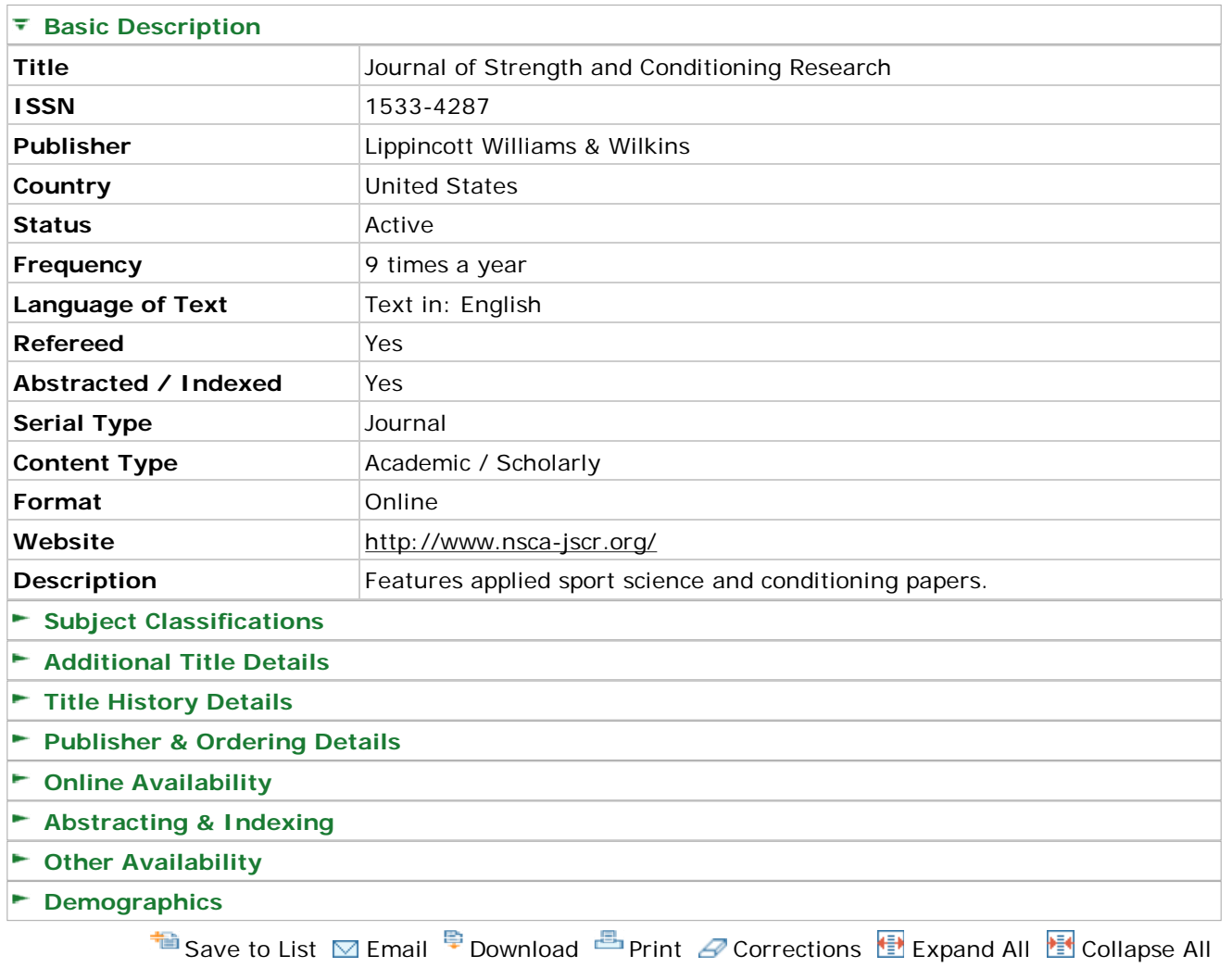

